\title{
Electronic Structure of Calcium Hexaborides
}

\author{
Byounghak Lee* and Lin-Wang Wang \\ Computational Research Division, Lawrence Berkeley \\ National Laboratory, Berkeley, California 94720 \\ (Received \\ )
}

\begin{abstract}
We present a theoretical study of crystal and electronic structures of $\mathrm{CaB}_{6}$ within a screenedexchange local density approximation (sX-LDA). Our ab initio total energy calculations show that $\mathrm{CaB}_{6}$ is a semiconductor with a gap of $>1.2 \mathrm{eV}$, in agreement with recent experimental observations. We show a very sensitive band gap dependence on the crystal internal parameter, which might partially explain the scatter of previous theoretical results. Our calculation demonstrates that it is essential to study this system simultaneously for both crystal structures and electronic properties, and that the sX-LDA provides an ideal method for this problem.
\end{abstract}

PACS numbers: 71.15.Mb, 71.20.Nr

${ }^{*}$ Electronic address: bhlee@lbl.gov 
The discovery of ferromagnetism in lightly doped $\mathrm{CaB}_{6}$ and $\mathrm{SrB}_{6}[1]$ has fueled intense investigation on these materials over the last several years. The remarkably high transition temperature $\left(T_{c} \approx 600 \mathrm{~K}\right)$ with a very low magnetic moment $\left(\leq 0.07 \mu_{B}\right.$ per atom $)$ has raised many speculations on the origin of the magnetism, including ferromagnetic phase within dilute electron gas [2] and doped excitonic insulators [3-5]. Although it now seems quite conclusive that the ferromagnetism is originated from the external impurities on the surface of the samples $[6,7]$ rather than from an intrinsic property of the system, the electronic band structure of the bulk systems is still under debate. While Fermi surface measurements [8] imply that $\mathrm{CaB}_{6}$ is a semimetal, angle-resolved photoemission spectroscopy (ARPES), momentum-resolved inelastic x-ray scattering [9], and transport [10] measurements indicate that $\mathrm{CaB}_{6}$ is a semiconductor with a gap larger than $1.0 \mathrm{eV}$. Theoretically, the local density approximation (LDA) has predicted a semimetallic band structure for $\mathrm{CaB}_{6}$ with a small overlap of boron-bands at the $\mathrm{X}$ point of the Brillouin zone [11-14], but it is well known that LDA calculations often underestimate the eigenenergy gap. On the other hand, more accurate many-body GW calculations have reported many different results. While a pseudopotential GW calculation led to a $0.8 \mathrm{eV}$ band gap [15] for $\mathrm{CaB}_{6}$, different full-potential LMTO GW calculations yielded 0.35 [16], -0.4 eV [17], and -0.7 eV [18].

One possible reason for the large differences in the theoretical results is the high sensitivity of the band gap to the crystal geometry parameters. Divalent hexaborides, including $\mathrm{CaB}_{6}$, have a simple cubic unit cell with metal ions $(\mathrm{Ca})$ located at the cubic corners and boron octahedral cages at the cubic center. The two relevant structural parameters are the lattice constant $a$ and the positional parameter $x$ of the boron atoms. The positions of boron atoms are $\pm\left(\frac{1}{2}, \frac{1}{2}, x\right), \pm\left(\frac{1}{2}, x, \frac{1}{2}\right), \pm\left(x, \frac{1}{2}, \frac{1}{2}\right)$ in the unit of lattice constant. When $x=0.207$, the intra-octahedral and inter-octahedral B-B distances are equal. As demonstrated below, the band gap of the system depends sensitively on the values of $a$ and $x$. Unfortunately, the experimentally measured results for $a$ and $x$ have not shown a complete agreement. The experimentally measured $\mathrm{CaB}_{6}$ lattice constant varies from 4.145 to $4.153 \AA$ [13], while LDA calculations predicts a lattice constant between 4.08 and $4.105 \AA$ [11, 13, 19]. The internal parameter $x$ has been observed experimentally from 0.200 to 0.207 [13] while LDA predictions vary from 0.201 to 0.2024 . We shall show that, within the experimental uncertainty of $x$, the calculated band gap can change by as much as $0.5 \mathrm{eV}$. This might partially explain the differences in the GW calculated band gaps. 
Due to the reasons discussed above, it is desirable to use a method which can both correct the LDA band gap error and calculate the total energy (thus can be used to study the geometry of the system). While the GW method is very accurate in describing the band structure, it cannot be used to calculate the total energy. Here, we use the screened exchange LDA (sX-LDA) method $[20,21]$ to study $\mathrm{CaB}_{6}$. sX-LDA is a variational total energy method with an explicit screened exchange energy designed to improve over LDA. It has been shown to accurately reproduce both band gap and structural properties of many simple semiconductor binary compounds [22]. However, it has not been tested thoroughly for more complicated systems such as $\mathrm{CaB}_{6}$ and the current study also serves as a test for sX-LDA itself.

In sX-LDA, the energy functional is modeled to include the nonlocal exchange-correlation hole within a generalized density functional scheme:

$$
E[\{\phi\}, n]=E_{L D A}[\{\phi\}, n]+E_{s x}^{N L}[\{\phi\}]-E_{s x}^{l o c}[n] .
$$

Here $E_{L D A}[\{\phi\}, n]$ is the usual LDA energy functional of electron density $n, E_{s x}^{N L}[\{\phi\}]$ is the nonlocal screened-exchange energy functional, and $E_{s x}^{l o c}[n]$ is a local density approximation of $E_{s x}^{N L}[\{\phi\}]$ to assure that sX-LDA leads to LDA in a homogeneous electron gas. We have used a simple Thomas-Fermi screening function for the Fock exchange energy:

$$
E_{s x}^{N L}[\{\phi\}]=-\frac{1}{2} \sum_{i, j}^{o c c} \int d \mathbf{r} d \mathbf{r}^{\prime} \frac{\phi_{i}^{*}(\mathbf{r}) \phi_{j}^{*}\left(\mathbf{r}^{\prime}\right) \phi_{j}(\mathbf{r}) \phi_{i}\left(\mathbf{r}^{\prime}\right) e^{-k_{T F}\left|\mathbf{r}-\mathbf{r}^{\prime}\right|}}{\left|\mathbf{r}-\mathbf{r}^{\prime}\right|},
$$

where $k_{T F}$ is the Thomas-Fermi wavelength determined from the average electron density. The use of the average electron density is not without justification. For example, it has been shown that the GW nonlocal self-energy operator in a semiconductor can be fit by the self-energy operator of jellium with the average density of the semiconductor [23]. We tested that the band structure does not sensitively depend on the screening length; For instance, upon a change in the average electron density used for the screening length by a factor of 2 , the sX-LDA band gaps for our system will differ only by $\approx 0.1 \mathrm{eV}$. Encouraging results have been observed in many semiconductor materials with Eq.(2), yielding accurate band gaps and structural properties, both better than LDA results [21, 22].

The calculations were done using a plane-wave basis with pseudopotentials. The integration over the Brillouin zone was done by sampling 20 special k-points, which is equivalent to 
$6 \times 6 \times 6 \mathrm{k}$-point grids. The kinetic energy cutoff is $60 \mathrm{Ry}$ and we used Troullier-Martins normconserving pseudopotentials. The variational solution of Eq.(1) is solved self-consistently.

We first study the band structure of this system. In order to compare the results of different methods, we choose a set of crystal parameters used in previous studies, $a=4.15$ $\AA$ and $x=0.207$, which is inside the range of experimental measurements. Our resulting LDA and sX-LDA band structures are shown in Fig. 1. The band gap appears at the X point. While there is a small LDA band gap of about $0.1 \mathrm{eV}$, the sX-LDA band gap is about $0.9 \mathrm{eV}$. Note the sX-LDA result is very close to the pseudopotential GW calculations using similar crystal structure parameters [15]. Besides the band gaps, our valence band width and the second and third subvalence band overlaps are also extremely close to the GW results as reported in Table I. This closeness of our sX-LDA results with the GW results indicates that the dynamic screening, which is missing in the sX-LDA formalism, might not be important here. We would also like to point out that a recent weighted density approximation (WDA) calculation by $\mathrm{Wu}$ et al. for the same system also produces a similar band gap [19]. The opened band gap is attributed to the corrected self-interaction in the WDA treatment. In our sX-LDA formalism, the erroneous self-interaction has also been corrected to some extent. This explains the increased band gap in our sX-LDA result and also the increased bond charge as will be discussed below.

The equilibrium crystal structure is found by minimizing the total energy in the two dimensional parameter space of $a$ and $x$. The sX-LDA relaxed minimum is at $a=4.15$ $\AA$ and $x=0.212$, while the LDA results are $a=4.11 \AA$ and $x=0.202$ as shown in Table I. The sX-LDA lattice constant is closer to the experimental results, while LDA underestimates it by $\approx 1 \%$ in accordance with previous calculations [11]. A more pronounced difference between LDA and sX-LDA predictions arises from the internal position of the B atoms. While LDA predicts a shorter inter-octahedral distance [A-C in Fig. 2 (b)] than the intra-octahedral bond length $b$ [A-B in Fig. 2 (b)], the sX-LDA predicts the opposite. The total charge density difference between the sX-LDA and LDA results is shown in Fig. 2(a). For comparison purpose, we used the same lattice constant, $4.15 \AA$, and internal parameter, 0.207, for both sX-LDA and LDA. Interestingly, the sX-LDA has more B-B bond charge both within the octahedra and between the octahedra. This is consistent with the results found in other conventional semiconductors, such as Si [21], where the enhancement of bonding charge is found in sX-LDA calculations and is consistent with experimental results [24]. 
This enhancement is caused by reducing the self-interaction effects within sX-LDA [25]. In equilibrium structures the difference in bond charge is even larger.

We have also investigated the bulk modulus and the optical phonon modes. One interesting optical phonon mode (breathing mode) corresponds to each B atom moving perpendicular to its nearest cubic planes. The frequency of this breathing mode is determined mostly by the strength of both the inter and intra B-B bonds. Our sX-LDA breathing mode frequency $\omega$ is $15 \%$ smaller than the LDA result (see Table I), indicating weaker B-B bonds and thus correcting the typical LDA overbinding. An interesting fact is that, as discussed above, the sX-LDA charge within the B-B bonding region is larger than the LDA result. This seemingly contradictory result is possible because the self-interaction of the bond charge in sX-LDA has been corrected, which allows more charge density at the bonds. Similarly, the sX-LDA bulk modulus is $3 \%$ smaller than the LDA result (see Table I), correcting the typical overestimation of LDA.

A deeper understanding of the band gap dependency on the crystal structure can be obtained by looking at the electron state wavefunctions. In Fig. 2 (b) and (c), we plotted in the real space the VBM and CBM wavefunctions (not their charge density) at the $\mathrm{X}$ point. The symmetry of LDA and sX-LDA orbitals are the same. We see that the VBM has a $t_{2 g}$ symmetry and that it consists of $\mathrm{B} p$ orbitals in the plane perpendicular to the $\mathrm{X}$-point. This is in agreement with the qualitative picture given by Greenwood et al [26]. In Ref. [26], metal cations were not considered and, as a result, the CBM consisted of B orbitals only, having $t_{2 u}$ symmetry. Our current calculation shows, to the contrary, that the CBM is composed of strongly hybridized B $p$ states and Ca $d$ states. We found that the total valence charge around the $\mathrm{Ca}$ atoms is almost zero. This indicates that each $\mathrm{Ca}$ atom donates 2 electrons to the B network. Fig. 3 shows the CBM and VBM band energy as a function of intra B-octahedron bond length $b$ at a fixed lattice constant $a$ of $4.15 \AA$. The band energy gap as well as the energy levels have a linear dependence on $b$. When the intra-octahedral B-B distances $b$ decreases, their bonding increases, thus lowering the energy of the VBM. The decrease in $b$ also decreases the overlap between the $\mathrm{Ca}$ and $\mathrm{B}$ atomic orbitals, which increases the CBM energy. Overall, the VBM is more sensitive to $b$ because it is located directly at the intra B-octahedron bond. We have shown in Fig. 4 the band gap dependence on the lattice constant $a$ within experimentally relevant range, while keeping the intra B-octahedron bond length $b$ fixed at $1.72 \AA$. The band gap has a 
linear dependence on $a$, similarly to the dependence on $b$. If $b$ is relaxed when $a$ changes, the dependence of the band gap on $a$ is still linear. However, the slope of the $E_{g}$ versus $a$ curve changes from $5.62(6.57) \mathrm{eV} / \AA$ (fixed $b$ ) to $2.31(3.47) \mathrm{eV} / \AA$ (relaxed $b$ ) in LDA (sX-LDA).

Finally, we discuss the dependence of the band gap on the equilibrium crystal structures. Using the LDA minimum energy crystal structure, we obtain a LDA band overlap of 0.32 $\mathrm{eV}$ as shown in Table I. At the sX-LDA equilibrium structure, the sX-LDA band gap is 1.27 $\mathrm{eV}$, in good agreement with the recent ARPES measurement [9]. Roughly, half of this large difference between LDA and sX-LDA results comes from the band gap difference of these two methods at a given crystal structure and the other half comes from their equilibrium crystal structure difference. As shown in Fig. 3 and Fig. 4, the band gap of this system is very sensitive to the crystal structure. Within the experimental uncertainties in $a$ and $b$, the band gap can easily change by $0.5 \mathrm{eV}$. This might partially explain the diversity of the previous theoretical results, e.g. of GW calculations. Thus, given the experimental uncertainties on the structure parameters, it is essential to study this problem with a method which can accurately predict both the crystal structure and electronic band gap. The sX-LDA provides with one such method.

In summary, we have used sX-LDA method to study the crystal structure parameters and band structure of the $\mathrm{CaB}_{6}$ system. We found that at the equilibrium crystal structure the band gap is $1.27 \mathrm{eV}$, in good agreement with recent experiments. Our sX-LDA result agrees well with the GW results for a given crystal structure. There is a sensitive dependence of the band gap on the crystal structure, which might explain the diversity of previous theoretical results. Two factors help the sX-LDA method correct the equilibrium LDA band gap; the first is to correct LDA overbinding and the second is to correct the self-interaction of the bond charge, which increases the band gap and the charge density at the B-B bond for a given crystal structure.

BL acknowledges J. Denlinger for inspiring discussions and T. Kotani for showing us his unpublished GW data. This work was supported by U.S. Department of Energy under contract No. DE-AC03-76SF00098 and the calculations were done using resources of NERSC at Lawrence Berkeley National Lab. 
[1] D. P. Young, D. Hall, M. E. Torelli, Z. Fisk, J. L. Sarrao, J. D. Thompson, H.-R. Ott, S. B. Oseroff, R. G. Goodrich, and R. Zysler, Nature (London) 397, 412 (1999).

[2] D. Ceperley, Nature (London) 397, 386 (1999).

[3] M. E. Zhitomirsky, T. M. Rice, and V. I. Anisimov, Nature (London) 402, 251 (1999).

[4] V. Barzykin and L. P. Gor'kov, Phys. Rev. Lett. 84, 2207 (2000).

[5] L. Balents and C. M. Varma, Phys. Rev. Lett. 84, 1264 (2000).

[6] K. Taniguchi, T. Katsufuji, F. Sakai, H. Ueda, K. Kitazawa, and H. Takagi, Phys. Rev. B 66, 064407 (2002).

[7] K. Matsubayashi, M. Maki, T. Moriwaka, T. Tsuzuki, T. Nishioka, C. H. Lee, A. Yamamoto, T. Ohta, and N. K. Sato, J. Phys. Soc. Jpn. 72, 2097 (2003).

[8] D. Hall, D. P. Young, Z. Fisk, T. P. Murphy, E. C. Palm, A. Teklu, and R. G. Goodrich, Phys. Rev. B 64, 233105 (2001).

[9] J. D. Denlinger, J. A. Clack, J. W. Allen, G.-H. Gweon, D. M. Poirier, C. G. Olson, J. L. Sarrao, A. D. Bianchi, and Z. Fisk, Phys. Rev. Lett. 89, 157691 (2002).

[10] B. K. Cho, J.-S. Rhyee, B. H. Oh, M. H. Jung, H. C. Kim, Y. K. Yoon, J. H. Kim, and T. Ekino, Phys. Rev. B 69, 113202 (2004).

[11] S. Massidda, A. Continenza, T. M. de Pascale, and R. Monnier, Z. Phy. B: Condens. Matter 102, 83 (1997).

[12] S. Massidda, R. Monnier, and E. Stoll, Eur. Phys. J. B 17, 645 (2000).

[13] K. Schmitt, C. Stuckl, H. Ripplinger, and B. Albert, Solid State Science 3, 321 (2001).

[14] C. O. Rodriguez, R. Weht, and W. E. Pickett, Phys. Rev. Lett. 84, 3903 (2000).

[15] H. J. Tromp, P. van Gelderen, P. J. Kelly, G. Brocks, and P. A. Bobbert, Phys. Rev. Lett. 87, 016401 (2001).

[16] M. van Schilfgaarde and T. Kotani (unpublished).

[17] H. Kino, F. Aryasetiawan, M. van Schilfgaarde, T. Kotani, T. Miyake, and K. Terakura, J. Phys. Chem. Solids 63, 1595 (2002).

[18] H. Kino, F. Aryasetiawan, K. Terakura, and T. Miyake, Phys. Rev. B 66, 121103 (2002).

[19] Z. Wu, D. J. Singh, and R. E. Cohen, Phys. Rev. B 69, 193105 (2004).

[20] D. M. Bylander and L. Kleinman, Phys. Rev. B 41, 7868 (1990). 
[21] A. Seidl, A. Görling, P. Vogl, J. A. Majewski, and M. Levy, Phys. Rev. B 53, 3764 (1996).

[22] C. B. Geller, W. Wolf, S. Picozzi, A. Continenza, R. Asahi, W. Mannstadt, A. J. Freeman, and E. Wimmer, Appl. Phys. Lett. 79, 368 (2001).

[23] R. W. Godby, M. Schlüter, and L. J. Sham, Phys. Rev. B 37, 10159 (1988).

[24] J. M. Zuo, P. Blaha, and K. Schwarz, J. Phys. Condens. Matter 9, 7541 (1997).

[25] R. Asahi, W. Mannstadt, and A. J. Freeman, Phys. Rev. B 59, 7486 (1999).

[26] N. N. Greenwood, R. V. Rarish, and P. Thornton, Chem. Soc. London, Q. Rev. 20, 441 (1996). 
TABLE I: The lattice constant $a$, the internal positional parameter $x$, the intra B-octahedron bond length $b=a \cdot x$, the bulk modulus $\mathrm{B}$, and the breathing mode frequency $\omega \cdot \mathrm{LDA}^{\dagger}\left(\mathrm{sX}-\mathrm{LDA}^{\dagger}\right)$ used $a=4.15 \AA$ and $x=0.207 . \mathrm{LDA}^{*}\left(\mathrm{sX}-\mathrm{LDA}^{*}\right)$ is the relaxed $a$ and $x$.

\begin{tabular}{ccccccc}
\hline \hline & $a(\AA)$ & $x$ & $b(\AA)$ & $\mathrm{B}(\mathrm{GPa})$ & $\omega\left(10^{14} \mathrm{~Hz}\right)$ & \\
\hline LDA & 4.11 & 0.202 & 1.73 & 151 & 2.35 & \\
sX-LDA & 4.15 & 0.212 & 1.69 & 146 & 2.04 & \\
\hline \hline & $\mathrm{LDA}^{\dagger}$ & $\mathrm{LDA}^{*}$ & $\mathrm{sX}^{-L^{\prime} A^{\dagger}}$ & $\mathrm{sX}-\mathrm{LDA}^{*}$ & $\mathrm{GW}[15]$ & Experiment [9] \\
\hline band gap $(\mathrm{eV})$ & 0.11 & -0.32 & 0.89 & 1.27 & 0.8 & $>1.15$ \\
band width $(\mathrm{eV})$ & 9.2 & 9.6 & 10.03 & 9.76 & 10 & 9.0 \\
2 and 3 valence band overlap $(\mathrm{eV})$ & 0.74 & 0.58 & 0.29 & 0.53 & 0.3 & 1.0 \\
\hline \hline
\end{tabular}


Fig. 1: Electronic band structure of $\mathrm{CaB}_{6}$ with experimental parameters; $a=4.15 \AA$ and $x=0.207$.

Fig. 2: (a) Valence electron density difference between LDA and sX-LDA with $a=4.15$ $\AA, x=0.207 ; \Delta n=n_{s X-L D A}(\mathbf{r})-n_{L D A}(\mathbf{r}) . \Delta n<0$ (blue). $\Delta n=0$ (yellow). $\Delta n>0$ (red). (b) The VBM wavefunction at X-point (in the $\mathrm{X}$-direction). Point $\mathrm{C}$ denotes a $\mathrm{B}$ atom from a neighboring octahedron. (c) The CBM wavefunction at X-point. In (b) and (c) the blue and red surface represent the iso-surface with positive and negative signs, respectively.

Fig. 3: X-point VBM and CBM energy levels as a function of intra B-octahedron bond length. The lattice constant is fixed at $4.15 \AA$. Both LDA and sX-LDA levels are rigidly shifted for the convenience of comparison. At $1.72 \AA$, the inter and intra B-octahedron distance become equal.

Fig. 4: Band energy gap at $\mathrm{X}$-point as a function of the lattice constant. The intra B-octahedron bond length is fixed at $1.72 \AA$. The open and closed symbols are the LDA and sX-LDA result, respectively. 


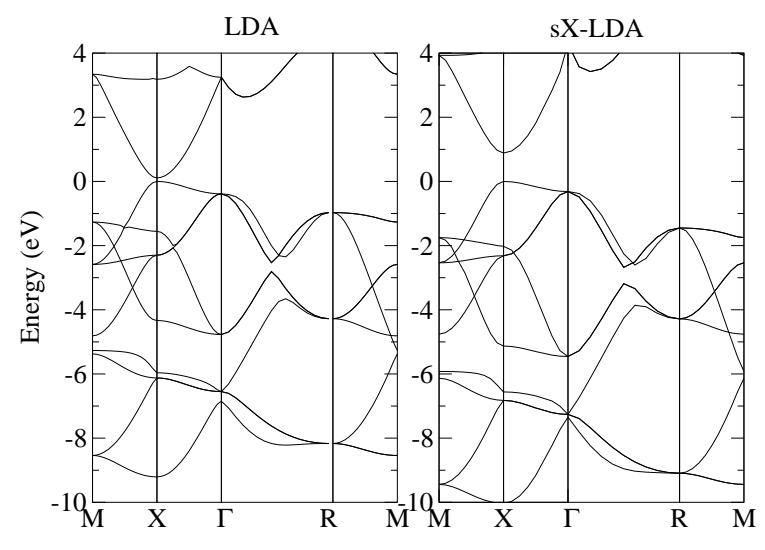

FIG. 1: 

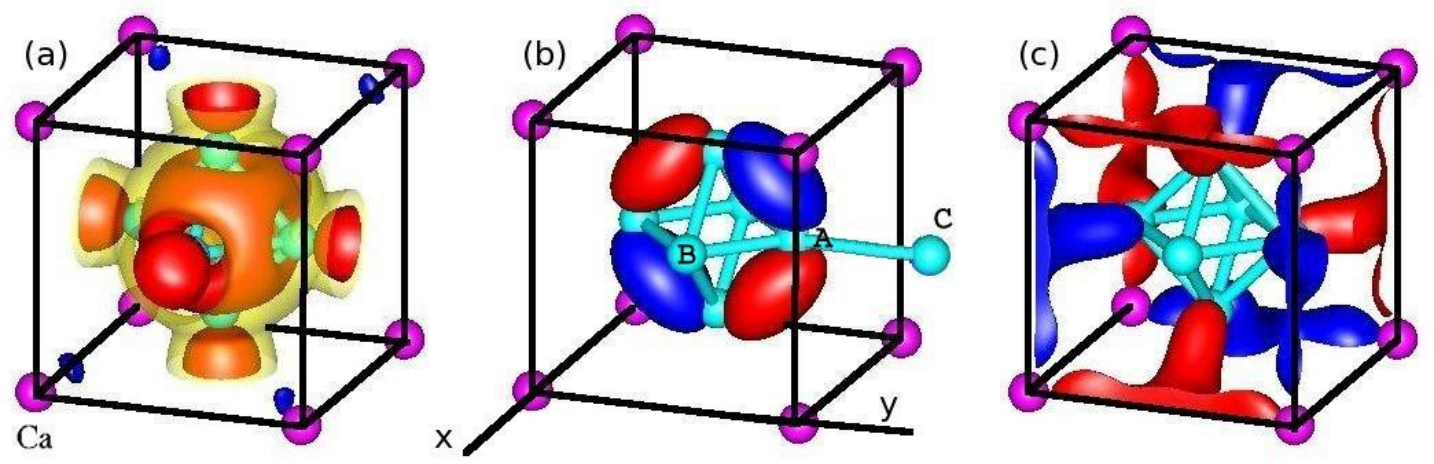

FIG. 2: 


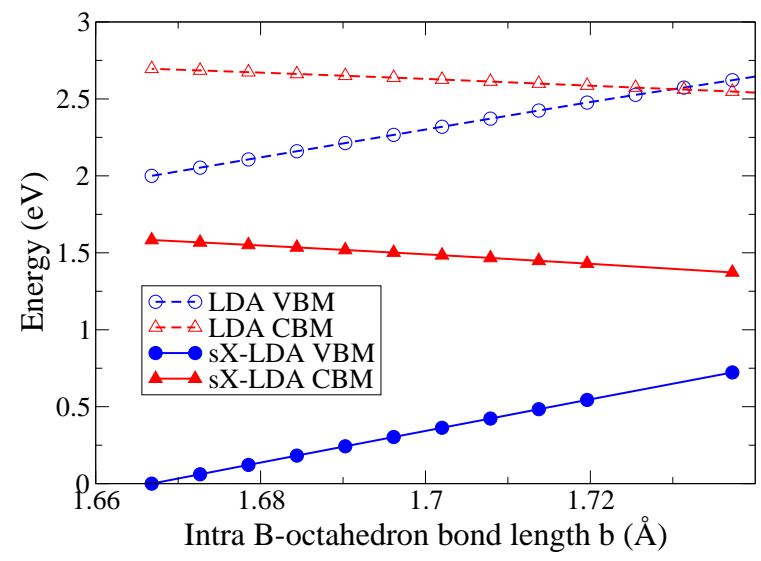

FIG. 3: 


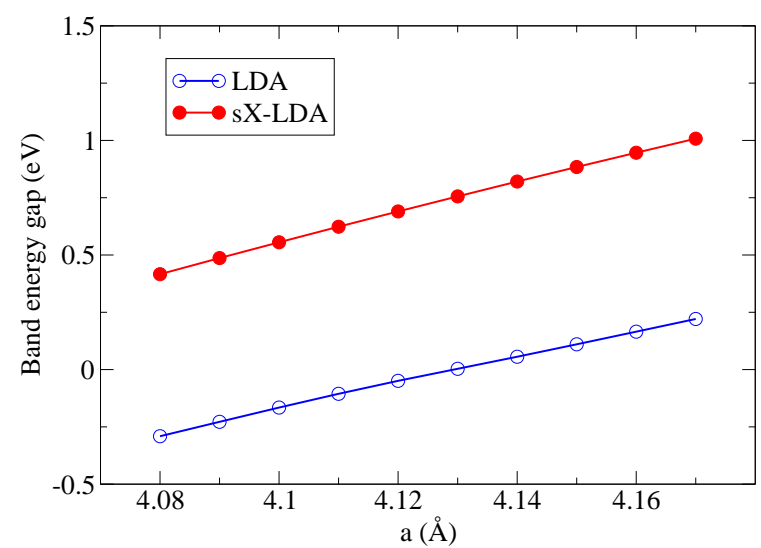

FIG. 4: 